\title{
A Constellation Space Dimensionality Reduced Sub-Optimal Receiver for Orthogonal STBC CPM Modulation in a MIMO Channel
}

\begin{abstract}
M. Hekrdla
We consider burst orthogonal space-time block coded (OSTBC) CPM modulation in a MIMO flat slow Rayleigh fading channel. The optimal receiver must process a multidimensional non-linear CPM signal on each antenna. This task imposes a high load on the receiver computational performance and increases its complexity. We analytically derive a suboptimal receiver with a reduced number of front end matched filters (MFs) corresponding to the CPM dimension. Our derivation is made fully in the constellation signal space, and the reduction is based on the linear orthogonal projection to the optimal subspace. Criterion optimality is a standard space-time rank and determinant criterion. The optimal arbitrary-dimensional subspace search leads to the eigenvector solution. We present the condition on a sufficient subspace dimension and interpret the meaning of the corresponding eigenvalues. It is shown that the determinant and rank criterion for OSTBC CPM is equivalent to the uncoded CPM Euclidean distance criterion. Hence the proposed receiver may be practical for uncoded $C P M$ and foremost in a serially concatenated (SC) CPM system. All the derivations are supported by suitable error simulations for binary $2 R E C h=1 / 2$, but the procedure is generally valid for any CPM variant. We consider OSTBC CPM in a Rayleigh fading AWGN channel and SC CPM in an AWGN channel.
\end{abstract}

Keywords: Space-Time Coding (STC), Continuous Phase Modulation (CPM), receiver complexity reduction, MIMO, burst Orthogonal Space-Time Block Coded (OSTBC) CPM, Serially Concatenated CPM (SCCPM), mismatched detector.

\section{Introduction}

\subsection{Background}

Continuous phase modulation (CPM) is a transmitterfriendly digital modulation scheme typically used in wireless communication systems e.g. Bluetooth, GSM. The constant envelope property allows the use of a low-cost, nonlinear power efficient amplifier typically from C class, whereas continuous phase forces the spectrum to decrease steeply and leads to spectral efficient modulation with a narrow bandwidth. However, in the typical wireless fading communication channel, uncoded CPM suffers from the same performance degradation as any linear modulation format. Hence, in the past few years, there has been an effort to extend the concept of space-time coding (STC), originally developed for linear modulations, to CPM. Several ST CPM coding schemes have been proposed that can be classified as ST trellis codes [20], [19]. These schemes have modified the phase trellis inherent to CPM, and therefore the existing CPM receiver schemes cannot be directly applied. These disadvantages do not have space-time block codes (STBC) [18]. Design rules combining orthogonality and CPM cannot be simply applied due to phase continuity violation. However, it might be easily accomplished with a burst-based approach [8]. Phase continuity among the bursts is ensured by the termination tail data sequence, which forces the modulator to the zero state at the end of each burst.

\subsection{Goals}

We propose an optimal reduced constellation subspace for burst OSTBC CPM with the optimality criterion given by a standard space-time determinant and rank criterion. We assume that the constellation basis fulfills orthogonality and the Nyquist condition in order to simplify metric computation of the receiver and prepare the ground for possible iterative detection. The well-known Laurent expansion [4], including its tilted forms variant, does not satisfy this. Receivers that use shorter phase pulses than the transmitter are called mismatched receivers [10]. This type of receivers are also used here. It is shown that the optimal subspace is described in the constellation space by eigenvectors of a special Hermitian matrix. This is formed by all signal vector differences making all minimal free paths of uncoded CPM, and depends only on the given CPM variant. The dimensionality of this space corresponds to the number of MFs practically implemented at each antenna of the receiver. The analytical derivation uses linear orthogonal subspace eigenvector projection in the constellation space. Suboptimal receivers are tested with binary 2REC $h=1 / 2$ in a $2 \times 1$ MIMO Rayleigh fading channel and the Alamouti space-time block code. The procedure itself has general validity for any space-time block code from the orthogonal design (OD) [16] and for any used CPM format.

\subsection{Prior art and related work}

Analytical projection to a one-dimensional subspace has been discussed in [14]. The procedure was based on earlier work with numerical optimization [13] and a definition of the optimality criterion [12]. The non-linear receiver preprocessor has been investigated in [11]. There has been a huge effort in CPM receiver complexity reduction. Papers dealing with this topic are [10], [3], [9], [15], [6] and references in them. 


\section{Definitions and system model}

\subsection{Tilted CPM definition}

For mathematical convenience we adopt the tilted phase CPM concept [7] with a time-invariant trellis and possibly reduced modulator states and distinct signal waveforms. A stationary trellis diagram is attractive for a minimum free distance search, the termination tail algorithm and a constellation basis search. The modulated bandpass complex envelope signal is defined as

$$
s(t, \boldsymbol{U})=\sqrt{\frac{2 \varepsilon_{s}}{T_{s}}} \mathrm{e}^{\left(j 2 \pi f_{1} t+\bar{\psi}(t, \boldsymbol{U})\right)}
$$

with shifted center carrier frequency $f_{1}=f_{0}-h\left(M_{d}-1\right) / 2 T_{s}$, where $T_{s}, M_{d}, \varepsilon_{s}, f_{0}$ is the symbol duration, alphabet size, symbol energy and original carrier frequency, respectively. The physically distinguishable phase is due to the harmonic function periodicity taken $\bar{\psi}=(\psi) \bmod 2 \pi$. The information-carrying tilted phase is

$$
\psi(t, \boldsymbol{d})=\phi(t, \boldsymbol{d})+\pi h(M-1) 1 / T_{s},
$$

where $\phi(t, \boldsymbol{d})$ is the original classic CPM phase. New data symbols $U_{n}$ take $M_{d}$ different values $U_{n} \in\left\{0,1, \ldots,\left(M_{d}-1\right)\right\}$. The physical phase function is $\bar{\psi}\left(\tau+n T_{s}, \boldsymbol{U}\right)=\left(2 \pi h V_{n}\right) \bmod 2 \pi$

$$
\begin{aligned}
& +4 \pi h \sum_{i=0}^{L-1} U_{n-i} \beta\left(\tau+i T_{s}\right) \\
& +W(\tau) ; \quad 0 \leq t<T_{s}, \quad n \geq L
\end{aligned}
$$

with the data-independent time-dependent part

$$
\begin{aligned}
W(\tau) & =\pi h\left(M_{d}-1\right) \frac{\tau}{T_{s}}-2 \pi h\left(M_{d}-1\right) \sum_{i=0}^{L-1} \beta\left(\tau+i T_{s}\right) \\
& +(L-1)\left(M_{d}-1\right) \pi h ; \quad 0 \leq \tau \leq T_{s} .
\end{aligned}
$$

The modulation index is a rational irreducible number $h=K / P$ and the alphabet size $M_{d}$ is assumed strictly power of 2 . The frequency pulse $\mu(t)$ with normalized area $\int_{-\infty}^{\infty} \mu(\tau) \mathrm{d} \tau=\frac{1}{2}$

defines the phase function $\beta(t)=\int_{-\infty}^{t} \mu(\tau) \mathrm{d} \tau$. Frequently-used pulses of the correlation length $L \in \mathbb{N}$ are

$$
\mu_{L R E C}(t)= \begin{cases}\frac{1}{2 L T_{s}}, & 0 \leq t \leq L T_{s}, \\ 0, & \text { else }\end{cases}
$$

$\mu_{L R C}(t)= \begin{cases}\frac{1}{2 L T_{S}}\left(1-\cos \frac{2 \pi t}{L T_{s}}\right), & 0 \leq t \leq L T_{s} . \\ 0, & \text { else }\end{cases}$

Modulator states are defined $\sigma_{n}=\left[U_{n-1}, \ldots, U_{n-L+1}, V_{n}\right]$. The accumulated state $V_{n}=\left(\sum_{i=0}^{n-L} U_{i}\right) \bmod P$ takes only $P$ distinct values, and so the modulator output signal is one of the $P M_{d}^{L}$ possible waveforms and the number of modulator states is equal to $P M_{d}^{(L-1)}$.The state equation for the accumulated phase follows $V_{n+1}=\left(V_{n}+U_{n-L+1}\right) \bmod P$.

\subsection{Constellation space}

We find the orthonormal and Nyquist basis by applying the Gram-Schmidt procedure to the maximum number of linearly independent modulator outputs $\left\{\xi_{j}(t)\right\}_{j=1}^{N_{s}}$ masked by a rectangular function. The orthonormal bases $\left\{\zeta_{i}(t)\right\}_{i=1}^{N_{s}}$ are obtained according to the Gram-Schmidt recursive formula

$$
\zeta_{j}(t)=\frac{\xi_{j}(t)-\sum_{i=1}^{j-1}\left\langle\xi_{j}(t), \zeta_{i}(t)\right\rangle \zeta_{i}(t)}{\left\|\xi_{j}(t)-\sum_{i=1}^{j-1}\left\langle\xi_{j}(t), \zeta_{i}(t)\right\rangle \zeta_{i}(t)\right\|},
$$

where $\langle\xi(t), \zeta(t)\rangle=\int_{0}^{T_{s}} \xi(t), \zeta^{*}(t) \mathrm{d} t$ is an inner product operation. Equivalently in matrix notation

$$
\boldsymbol{A}\left[\begin{array}{c}
\zeta_{1}(t) \\
\zeta_{2}(t) \\
\vdots \\
\zeta_{N_{S}}(t)
\end{array}\right]=\left[\begin{array}{c}
\xi_{1}(t) \\
\xi_{2}(t) \\
\vdots \\
\xi_{N_{S}}(t)
\end{array}\right]
$$

where matrix

$$
\boldsymbol{A}=\left[\begin{array}{cccc}
c_{1} & 0 & \cdots & 0 \\
a_{21} & c_{2} & \cdots & 0 \\
\vdots & \vdots & \ddots & \vdots \\
a_{N_{S} 1} & a_{N_{S} 2} & \cdots & c_{N_{S}}
\end{array}\right],
$$

with $\quad a_{i j}=\left\langle\xi_{j}(t), \zeta_{i}(t)\right\rangle$ and $\quad c_{j}=\left\|\xi_{j}(t)-\sum_{i=1}^{j-1}\left\langle\xi_{j}, \zeta_{i}\right\rangle \zeta_{i}(t)\right\|$. The modulation dimension is denoted $N_{s}$. The maximum number of linearly independent outputs can be obtained for $V_{n}=0$, because the other functions are a multiple of $\exp \left(j 2 \pi h V_{n}\right)$. This determines that the corresponding constellation vectors are directly equal to the individual rows of matrix $\boldsymbol{A}$ and the other constellation vectors are multiplied by $e^{j 2 \pi h V n}$.

Note that the modulator output waveforms for the case of REC are linearly dependent for the same data permutation. The final shape of the output signal is given by the sum of the data sequence of length $L$. In that case, dimension $N_{S}$ is equal to the number of all possible different summation results. The summation can take any number from 0 to $L\left(M_{d}-1\right)$, hence

$$
N_{s}^{R E C}=L\left(M_{d}-1\right)+1 .
$$

Considering RC and other similarly piecewise linearly independent pulses we cannot use the previous trick and hence

$$
N_{s}^{R C}=M_{d}^{L} \text {. }
$$




\subsection{Burst orthogonal space-time block coded CPM}

Without loss of generality we consider the only one full rate Alamouti scheme (transmit antennas $N_{T}=2$ ) as the most important representative of OSTBC. We consider signal division corresponding to the two $N_{d} / 2$ data messages, where $N_{d}$ denotes the number of data symbols in the message. Let us define $s_{1}(t)=s(t)$ for $0 \leq t<T_{s} N_{d} / 2$ and zero otherwise, and similarly $s_{2}(t)=s\left(t+T_{s} N_{d} / 2\right) ; 0 \leq t<T_{s} N_{d} / 2$. The resulting space-time $N_{T} \times N_{d} T_{s}$ continuous-time signal

$$
\boldsymbol{s}(t)=\left[\begin{array}{cc}
s_{1}(t) & -s_{2}^{*}(t) \\
s_{2}(t) & s_{1}^{*}(t)
\end{array}\right],
$$

can be described in the constellation space with

$s(t) \sim\left[s_{1}, s_{2}, \ldots, s_{N_{d}}\right]$,

$s_{1}(t) \sim\left[s_{1}, s_{2}, \ldots, s_{N_{d} / 2}\right]$ and

$s_{2}(t) \sim\left[\boldsymbol{s}_{N_{d} / 2+1}, \ldots, \boldsymbol{s}_{N_{d}}\right]$

by the space-time codeword matrix [18]

$\boldsymbol{S}=\left[\begin{array}{cccccc}\boldsymbol{s}_{1}^{T} & \ldots & \boldsymbol{s}_{N_{d} / 2}^{T} & -\boldsymbol{s}_{N_{d} / 2+1}^{H} & \ldots & -\boldsymbol{s}_{N_{d}}^{H} \\ \boldsymbol{s}_{N_{d} / 2+1}^{T} & \ldots & \boldsymbol{s}_{N_{d}}^{T} & \boldsymbol{s}_{1}^{H} & \ldots & \boldsymbol{s}_{N_{d} / 2+1}^{H}\end{array}\right]$

The block structure may cancel important phase continuity. Due to the long blocks the continuity violation will result in a small spectrum broadening, but long blocks lead to long delays in communication. So we use the termination tail technique, which forces the CPM modulator to the well-defined finish state at the end of each block and preserves the phase continuity. In addition, it increases the minimal free dis tance, but it also leads to some bits that carry no information [8]. The maximal termination tail length in symbols is obtained considering $U_{n-1} \oplus U_{n-2} \oplus \ldots \oplus U_{n-L+1} \oplus V_{n}=1$ as

$$
N_{t t}=\left\lceil\frac{P-1}{M_{d}-1}\right\rceil+L-1,
$$

$\oplus$ denotes modulo $P$ addition and $\lceil *\rceil$ is an integer part rounded up.

\subsection{Channel model}

A MIMO system model in constellation space describes received signal at the $k^{\text {th }}$ antenna for $t \in\left(n T_{s},(n+1) T_{s}\right)$ as

$$
\boldsymbol{x}_{n, k}=\sum_{i=1}^{N_{T}} h_{k i} \boldsymbol{s}_{n, i}+\boldsymbol{w}_{n, k} \text {. }
$$

In the block constant flat Rayleigh fading channel, the coefficients $h_{k i}$ are complex Gaussian zero mean independent identically distributed (IID) random variables with unity variance. Additive white Gaussian noise (AWGN) is represented by vector $w$. It is a complex random zero mean Gaussian variable with power $2 \boldsymbol{N}_{0}$ per dimension.

\section{Optimal projection subspace}

\subsection{Rank and determinant criterion for STC in flat Rayleigh fading channel}

The design criteria for space-time coding in quasi-static fading were originally designed to optimize the worst case pairwise error probability (PWEP) [17]. If we denote $\Delta \boldsymbol{S}=\boldsymbol{S}\left(\boldsymbol{d}^{(i)}\right)-\boldsymbol{S}\left(\boldsymbol{d}^{(k)}\right), i \neq k$ as a two distinct codeword difference and codeword distance matrix $\boldsymbol{R}=\Delta \boldsymbol{S} \Delta \boldsymbol{S}^{H}$

1. The code design rule for $N_{T} N_{R}<4$ follows.

(a) Maximize the minimum rank $r$ of matrix $\boldsymbol{R}$ and

(b) maximize the minimum product $\prod_{i} \lambda_{i}$ for all nonzero $\lambda_{i}$ over all pairs of distinct codeword differences $\Delta \boldsymbol{S}$.

2. For the case $N_{T} N_{R} \geq 4$,

(a) make sure that the minimum rank $r$ is such that $r N_{R} \geq 4$ and

(b) maximize the minimum trace $\sum_{i} \lambda_{i}$ of matrix $\boldsymbol{R}$ among all pairs of distinct codeword differences $\Delta S$.

The minimum operation is over all distinct codeword pairs and maximization should be accomplished with suitable STC.

Burst Alamouti coded CPM codeword difference matrix is according to (12)

$\Delta \boldsymbol{S}=\left[\begin{array}{cccccc}\Delta \boldsymbol{s}_{1}^{T} & \ldots & \Delta \boldsymbol{s}_{N_{d} / 2}^{T} & -\Delta \boldsymbol{s}_{N_{d} / 2+1}^{H} & \ldots & -\Delta \boldsymbol{s}_{N_{d}}^{H} \\ \Delta \boldsymbol{s}_{N_{d} / 2+1}^{T} & \ldots & \Delta \boldsymbol{s}_{N_{d}}^{T} & \Delta \boldsymbol{s}_{1}^{H} & \ldots & \Delta \boldsymbol{s}_{N_{d} / 2}^{H}\end{array}\right]$.

The codeword distance matrix is then simplified to a diagonal matrix

$$
\boldsymbol{R}=\left(\sum_{i=1}^{N_{d}}\left\|\Delta \boldsymbol{s}_{i}\right\|^{2}\right) \boldsymbol{I}_{N_{T}}
$$

We may expect this result, since OSTBC keeps orthogonality between signals transmitted from each antennas. Hence all its eigenvalues are equal $\lambda_{1}=\lambda_{2}=\ldots=\lambda_{N_{T}}=\lambda$ and so this scheme has a full rank and determinant equal to $\operatorname{det}(\boldsymbol{R})=\prod_{i=1}^{N_{T}} \lambda_{i}=\lambda^{N_{T}}$

Note that the eigenvalues of diagonal distance matrix $\boldsymbol{R}$ are equal to the Euclidean distance of uncoded CPM between $s^{(i)}(t)$ and $s^{(k)}(t)$.

$$
\lambda=\sum_{i=1}^{N_{d}}\left\|\Delta s_{i}\right\|^{2}=d_{i k}^{2}
$$

Both the rank\&determinant and the rank\&trace criterion are equivalent to the minimal free distance of uncoded CPM 
maximization. Assuming that the minimal $d_{\min }^{2}$ in most influences the error rate performance for medium to high SNR, we have to find a subspace reducing projection that preserves these minimal free distances. We may observe that the suboptimal receiver would also be optimal for uncoded CPM.

\subsection{Minimal energy error events}

The minimal free distance for a general particular response $(L>1)$ CPM is commonly obtained numerically [2, 1]. Because we assume zero start and termination state, we may conclude that free distances are all trellis paths that split and merge and are otherwise equal. The set containing all signal differences forming all minimal free distances $\Delta=\left\{\Delta \boldsymbol{s}_{1}, \ldots, \Delta \boldsymbol{s}_{N_{\Delta}}\right\}$ has $N_{\Delta}$ components. For example, binary 2REC $h=1 / 2$ with dimension $N_{S}=3$ has the free paths shown by bolted lines in Fig. 1. In the minimal path search it is sufficient to consider signals with $V_{n}=0$, due to CPM rotational symmetry.

\subsection{Optimal linear orthogonal subspace projection}

According to the STC code construction rules, we search for a projection subspace where the energy of minimal CPM path differences is maximal. In other words, we search for the best approximation of minimal Euclidean differences with a lower-dimensional signal. The basic proper ties of linear projectors [5] are $\boldsymbol{P}=\boldsymbol{B}\left(\boldsymbol{B}^{H} \boldsymbol{B}\right)^{-1} \boldsymbol{B}^{H}, \boldsymbol{P}^{H}=\boldsymbol{P}, \boldsymbol{P}^{2}=\boldsymbol{P}$, where matrix $\boldsymbol{B}$ consists of the projection subspace generally linearly independent basis $\boldsymbol{B}=\left[\boldsymbol{b}_{1}, \boldsymbol{b}_{2}, \ldots, \boldsymbol{b}_{N_{P}}\right]$. Our task is to find such $\boldsymbol{B}$ that preserves all minimal free distances

$$
\hat{\boldsymbol{B}}=\arg \max _{\breve{\boldsymbol{B}}}\left(\sum_{i=1}^{N_{\Delta}}\left\|\boldsymbol{P}(\breve{\boldsymbol{B}}) \Delta \boldsymbol{s}_{i}\right\|^{2}\right) .
$$

We should note that the projection focusing minimal distances will also change the overall distance spectrum. Thus after the projection e.g. the second minimum might be lower than the first minimum resulting in not-optimal performance. Hence the general joint optimization problem

$$
\hat{\boldsymbol{B}}=\arg \max _{\breve{\boldsymbol{B}}} \min _{\Delta \boldsymbol{s}}\left(\sum_{i=1}^{N_{\Delta}}\left\|\boldsymbol{P}(\breve{\boldsymbol{B}}) \Delta \boldsymbol{s}_{i}\right\|^{2}\right)
$$

should be assumed. Due to computational tractability, we assume that the minimal error event will also be minimal after the projection, and confirm this condition afterwards.

The projection to $N_{p}$ dimensional subspace along the orthonormal base function set $\left\{\boldsymbol{b}_{1}, \boldsymbol{b}_{2}, \ldots, \boldsymbol{b}_{N_{p}}\right\}$ is defined by the projector $\boldsymbol{P}=\boldsymbol{B} \boldsymbol{B}^{H}$. The term in (22) simplifies

$\sum_{i=1}^{N_{\Delta}}\left\|\boldsymbol{P} \Delta \boldsymbol{s}_{i}\right\|^{2}=\sum_{i=1}^{N_{\Delta}} \Delta \boldsymbol{s}_{i}^{H} \boldsymbol{P} \Delta \boldsymbol{s}_{i}=\sum_{j=1}^{N_{p}} \sum_{i=1}^{N_{\Delta}} \boldsymbol{b}_{j}^{H} \Delta \boldsymbol{s}_{i} \Delta \boldsymbol{s}_{i}^{H} \boldsymbol{b}_{j}$.

The optimization problem is then

$$
\sum_{j=1}^{N_{p}} \boldsymbol{b}_{j}^{H} \boldsymbol{Q} \boldsymbol{b}_{j} \stackrel{!}{=} \max
$$

where the sum of all outer products

$$
\boldsymbol{Q}=\left(\sum_{i=1}^{N_{\Delta}} \Delta \boldsymbol{s}_{i} \Delta \boldsymbol{s}_{i}^{H}\right)
$$

The sum of $N_{p}$ real non-negative quadratic forms (19) is maximized when each element is maximized. Now we employ the spectral theorem on condition that $\boldsymbol{b}_{1}, \boldsymbol{b}_{2}, \ldots, \boldsymbol{b}_{N p}$ must form an orthonormal set.

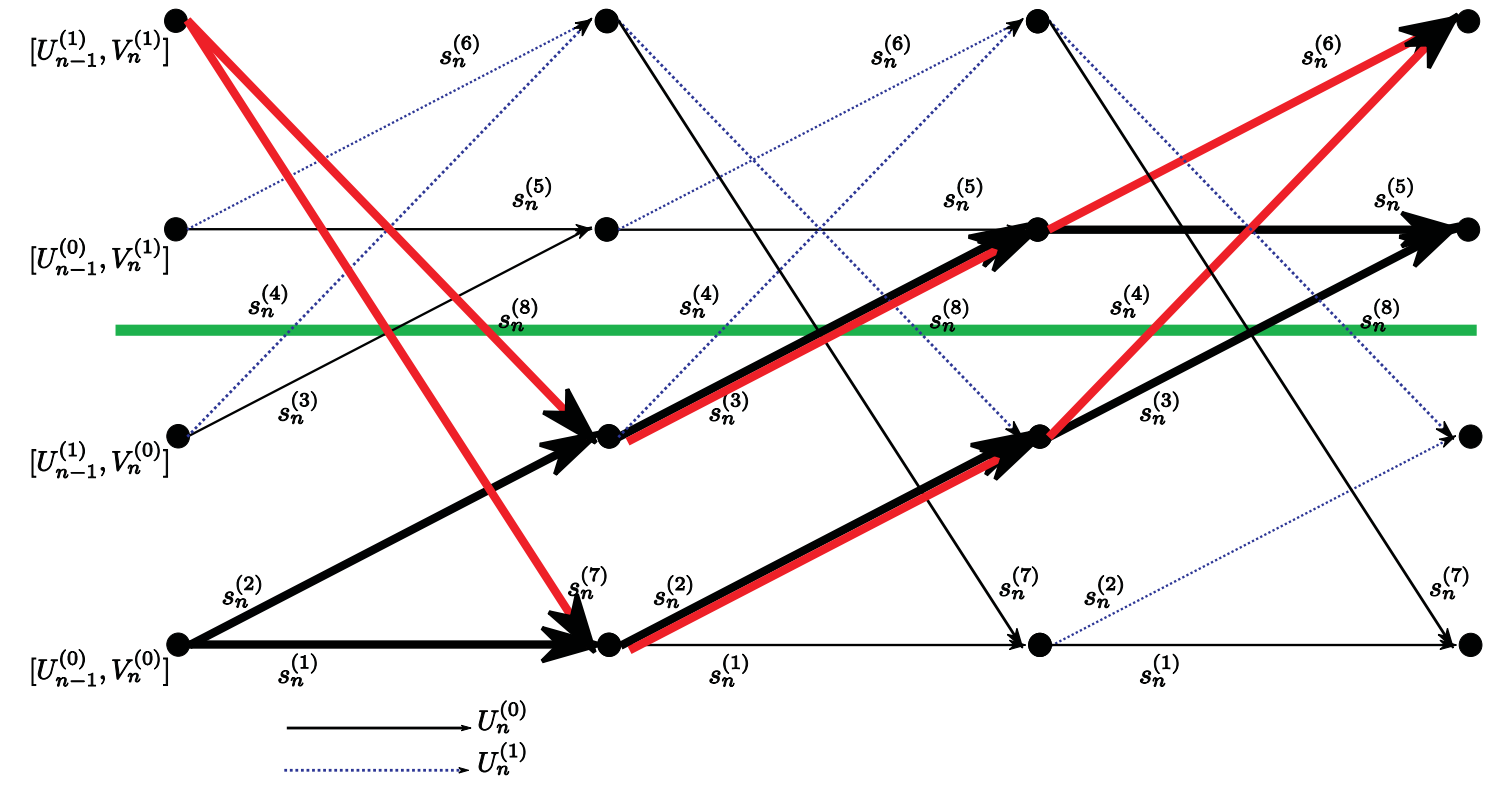

Fig. 1: Binary 2REC $h=1 / 2$ minimal free paths (thick lines) 
The spectral theorem of quadratic form [5] says $\lambda_{\min } \leq \boldsymbol{x}^{H} \boldsymbol{A} \boldsymbol{x} \leq \lambda_{\max } ; \forall \boldsymbol{x}:\|\boldsymbol{x}\|^{2}=1$

for $\boldsymbol{A}$ hermitian, where $\lambda$ is an eigenvalue of $\boldsymbol{A}$.

Here the conditions are fulfilled since the matrix $\boldsymbol{Q}^{H}=\boldsymbol{Q}$ is hermitian and $\left\|\boldsymbol{b}_{j}\right\|^{2}=1$. Thus

$$
\boldsymbol{b}_{j}^{H} \boldsymbol{Q} \boldsymbol{b}_{j} \leq \lambda_{\max } .
$$

For a subspace basis equal to unit $\boldsymbol{Q}$ eigenvectors $\boldsymbol{v}_{i}$, the quadratic form $\boldsymbol{v}_{i}^{H} \boldsymbol{Q} \boldsymbol{v}_{i}=\boldsymbol{v}_{i}^{H} \lambda_{i} \boldsymbol{v}_{i}=\lambda_{i}\left\|\boldsymbol{v}_{i}\right\|^{2}=\lambda_{i}$ and similarly $\boldsymbol{v}_{\max }^{H} \boldsymbol{Q} v_{\max }=\lambda_{\max }$. Considering (21), we have found the maximum. Assuming all eigenvalues are decreasing $\lambda_{1} \geq . . \geq \lambda_{N_{s}}$ the optimal projection basis is

$$
\hat{\boldsymbol{B}}=\left[v_{1}, \ldots, v_{N_{p}}\right] \text {. }
$$

The minimal free distance after the projection is equal to $\sum_{i=1}^{N_{\Delta}} \lambda_{i}$. Considering $\boldsymbol{P}=\boldsymbol{I}_{N_{s}}$ we conclude that the sum of all eigenvalues is equal to the total signal energy

$\sum_{i=1}^{N_{\Delta}}\left\|\Delta \boldsymbol{s}_{i}\right\|^{2}=\sum_{i=1}^{N_{s}} \lambda_{i}$.

Therefore, the error energy of the difference between a signal and its approximation is

$$
\sum_{i=N_{p}+1}^{N_{s}} \lambda_{i}
$$

As we discussed above, if we found all minimal free distances for zero accumulated metric $V_{n}=0$, we would obtain the rest of all other minimal free paths simply by a shift of $\mathrm{e}^{j 2 \pi V_{n}}$. Comparing matrix $\boldsymbol{Q}$, which we get from all signal differences forming all free distances, and matrix $\boldsymbol{Q}^{\prime}$, which consists of those with $V_{n}=0$, we conclude that the eigenvectors are the same, because

$\Delta \boldsymbol{s} \Delta \boldsymbol{s}^{H}=\mathrm{e}^{j 2 \pi V_{n}} \Delta \boldsymbol{s}^{\prime} \mathrm{e}^{-j 2 \pi V_{n}} \Delta \boldsymbol{s}^{\boldsymbol{H}^{H}}=\Delta \boldsymbol{s}^{\prime} \Delta \boldsymbol{s}^{\prime H}$.

For this reason, the eigenvectors of matrix $\boldsymbol{Q}$ are fully described for free paths with $V_{n}=0$, and so $N_{\Delta}$ depends only on $L$ and $M_{d}$ (not a function of $h$ ).

\subsection{Sub-optimal receiver basis}

It can be shown, that the implemented subspace basis functions are obtained from the optimal basis in constellation space (22) and the original orthonormal bases $\left\{\zeta_{i}\right\}_{i-1}^{N_{s}}$ according to the following formula.

$$
\varphi_{i}(t)=\boldsymbol{v}_{i}^{T}\left[\begin{array}{c}
\zeta_{1}(t) \\
\vdots \\
\zeta_{N_{s}}(t)
\end{array}\right], \text { for } i=1, \ldots, N_{p} .
$$

\section{Numerical Results}

For error performance verification we chose binary 2REC $h=1 / 2$ with free error events, depicted in Fig. 1, and we assume a $2 \times 1$ MIMO Rayleigh fading channel with burst Alamouti STBC CPM. According to the notes at the end of sec. 3.2 and 3.3, we need to investigate the trellis only for $V_{n}=0$. There are 4 minimal free paths with distance $d_{\text {min }}^{2}=3.454$ that consist of vectors

$$
\Delta=\left\{\boldsymbol{d}_{12}, \boldsymbol{d}_{78}, 2 \boldsymbol{d}_{23}, \boldsymbol{d}_{35}, \boldsymbol{d}_{46}\right\},
$$

where the constellation vector difference is $\boldsymbol{d}_{i k}=\boldsymbol{s}^{(i)}-\boldsymbol{s}^{(k)}$. The resulting matrix

$\boldsymbol{Q}=\boldsymbol{d}_{12} \boldsymbol{d}_{12}^{H}+\boldsymbol{d}_{78} \boldsymbol{d}_{78}^{H}+\boldsymbol{d}_{23} \boldsymbol{d}_{23}^{H}+\boldsymbol{d}_{35} \boldsymbol{d}_{35}^{H}+\boldsymbol{d}_{46} \boldsymbol{d}_{46}^{H}$.

The corresponding eigenvalues are

$\left\{\lambda_{1}, \lambda_{2}, \lambda_{3}\right\}=\{0.015,0.363,3.075\}$. Hence the distance spectrum changes negligibly (by 0.015) after projection along $\left\{\boldsymbol{v}_{2}, \boldsymbol{v}_{3}\right\}$, see Fig. 2. Abbreviations 'no', 'v3', 'v2v3' mean re-

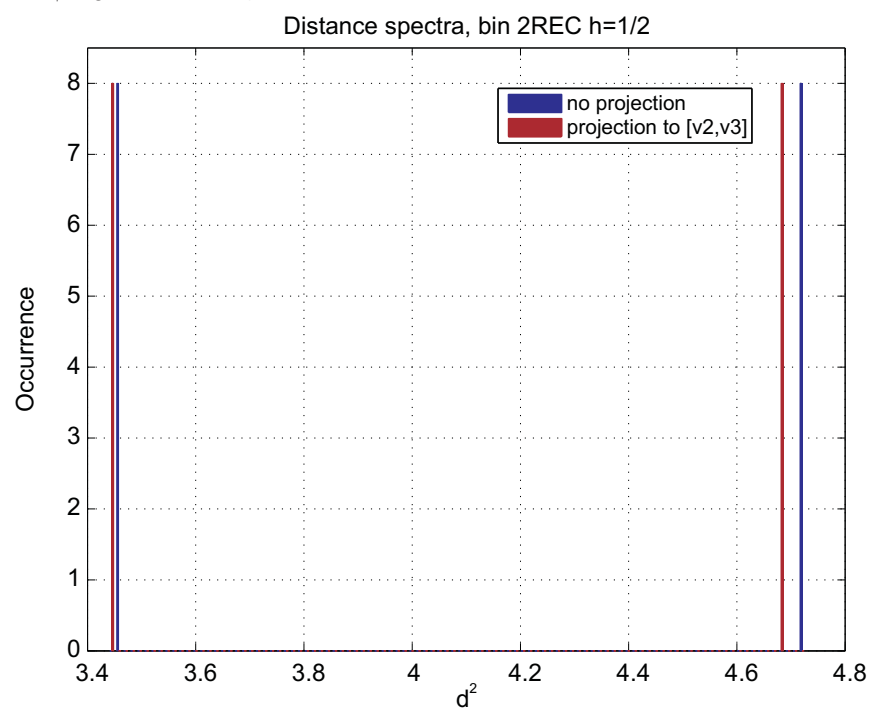

Fig. 2: Bin 2REC $h=1 / 2$ distance spectrum for error length 3

ceiver without dimensionality reducing projection, suboptimal receiver with projection to the subspace given by $\boldsymbol{v}_{3}$ and

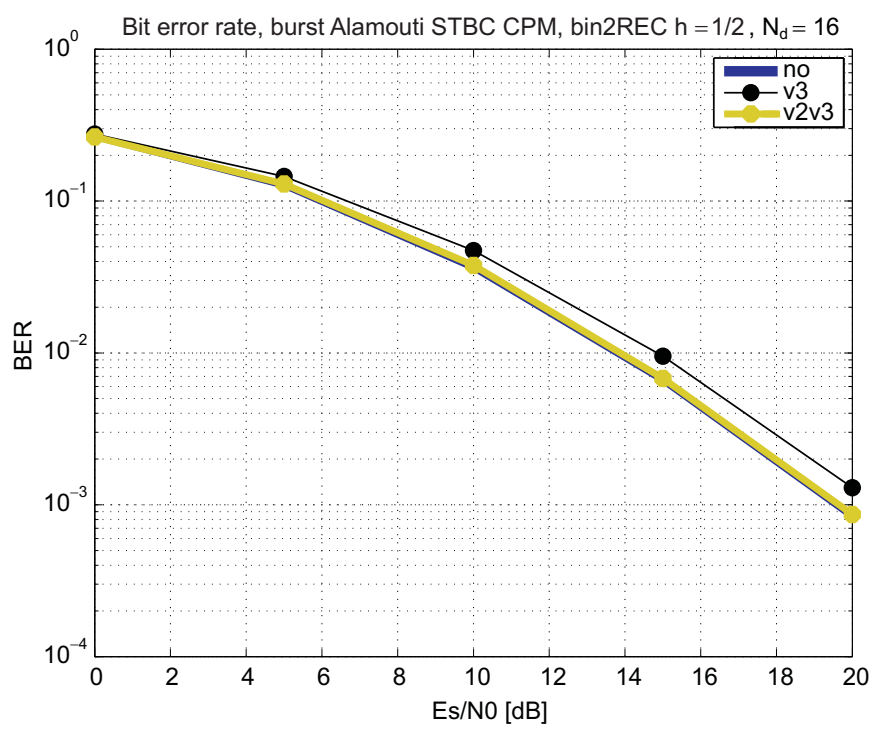

Fig. 3: Burst Alamouti STBC Bin 2REC $h=1 / 2$ error rate performance in a Rayleigh fading channel 
by $\left\{\boldsymbol{v}_{2}, \boldsymbol{v}_{3}\right\}$, respectively. The error rate performance is shown in Fig. 3. An interesting simulation was performed for serially concatenated bit random interleaved $(5,7)$ convolution code in the AWGN channel, see Fig. 4.

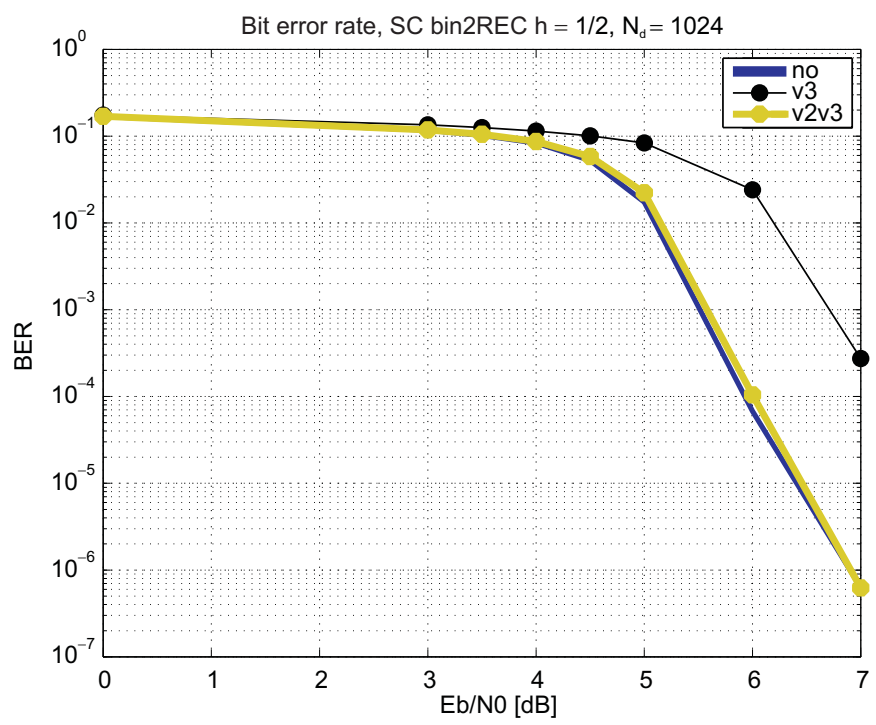

Fig. 4: SC Bin 2REC $h=1 / 2$ error rate performance in the AWGN channel

\section{Conclusions and Discussion}

We have analytically found a procedure for designing a suboptimal reduced dimensionality receiver for burst OSTBC CPM, based on the standard space-time code construction rules. We observed in the derivations that this procedure can also be applied to the suboptimal receiver for uncoded CPM. The derivation was realized fully in the constellation space based on linear orthogonal projection. The whole problem leads to the eigenvector solution, where the corresponding eigenvalues agree with the energy distribution of the minimal free distance to its orthogonal eigenvector subspaces. The true values of these eigenvalues for given CPM describe possible dimensionality reduction with no impact on performance. It has been shown that for minimal free paths and an optimal eigenvector search it is sufficient to consider the zero accumulated phase $V_{n}=0$. The suggested procedure was simulated for a specific CPM variant - binary 2REC $h=1 / 2$ with a result corresponding to the analytical solution.

\section{Acknowledgment}

I wish to thank Prof. Ing. Jan Sýkora, CSc., who gave me the opportunity to work on up-to-date research in cutting edge technology, gave me full support and started my professional growth. I have enjoyed close collaboration with him as well as with other members of the Digital Radio Communication (DiRac) Group.

\section{References}

[1] Aulin, T., Rydbeck, N., Sundberg, C-E. W.: Continuous Phase Modulation Part II: Partial Response Signaling. Communications, IEEE Transactions on, Vol. 29 (1981), No. 3, p. 210-225.
[2] Aulin, T., Sundberg, C-E. W.: Continuous Phase Modulation Part I: Full Response Signaling. Communications, IEEE Transactions on, Vol. 29 (1981), No. 3, p. 196-209.

[3] Huber, J., Liu, W.: An Alternative Approach to Reduced Complexity CPM Receivers. IEEE Journal on Selected Areas in Communications, Vol. 7 (1989), p. 1427-1436.

[4] Laurent, P. A.: Exact and Approximate Construction of Digital Phase Modulations by Superposition of Amplitude Modulated Pulses (AMP). IEEE Trans. Commun., COM, Vol. 34(1986), No. 2, p. 150-160.

[5] Meyer, C. D.: Matrix Analysis and Applied Linear Algebra. SIAM, 2000.

[6] Moqvist, P., Aulin, T.: Orthogonalization by Principal Components Applied to CPM. IEEE Transactions on Communications, Vol. 51(2003), p. 1838-1845.

[7] Rimoldi, B. E.: A Decomposition Approach to CPM. IEEE Trans. Information Theory, Vol. 34 (1988), No. 2, p. 260-270.

[8] Silvester, A. M., Schober, R., Lampe, L.: Burst-Based Orthogonal ST Block Coding for CPM. In Proc. IEEE Global Telecommunications Conference (GlobeCom), 2005.

[9] Simmons, S. J.: Simplified Coherent Detection of CPM. Communications, IEEE Transactions on, Vol. 43 (1995), No. 2,3,4, p. 726-728.

[10] Svensson, A., Sundberg, C.-E., Aulin, T.: A Class of Reduced Complexity Viterbi Detectors for Partial Response Continuous Phase Modulation. Communications, IEEE Transactions on, Vol. 32 (1984), p. 1079-1087.

[11] Sykora, J.: Information Waveform Manifold Based Preprocessing for Nonlinear Multichannel Modulation in MIMO Channel. In Proc. IEEE Global Telecommunications Conference (GlobeCom), St. Louis, USA, November 2005, p. 1-6.

[12] Sykora, J.: Linear Subspace Projection and Information Waveform Manifold Based Preprocessing for Nonlinear Multichanel Modulation in MIMO Channel. In Proc. Int. Conf. on Telecommunications (ICT), Cape Town, South Africa, May 2005. Invited paper, p. 1-6.

[13] Sykora, J.: Receiver Constellation Waveform Subspace Preprocessing for Burst Alamouti Block STC CPM Modulation. Proc. IEEE Wireless Commun. Network. Conference (WCNC), March 2007, p. 1-5.

[14] Sykora, J., Hekrdla, M.: Determinant Criterion Optimizing Linear Subspace Projector for Burst Orthogonal STC CPM Modulation in MIMO Channel. IEEE VTC, Spring 2009.

[15] Tang, W., Shwedyk, E.: A Quasi-Optimum Receiver for Continuous Phase Modulation. IEEE Transactions on Communications, Vol. 48 (2000), p. 1087-1090.

[16] Tarokh, V., Jafarkhani, H., Calderbank, A. R.: Space-Time Block Codes from Orthogonal Designs. Information Theory, IEEE Transactions on, Vol. 45 (1999), No. 5, p. 1456-1467.

[17] VTarokh, V., Seshadri, N., Calderbank, A. R.: Space-Time Codes for High Data Rate Wireless Communication: Performance Criterion and Code Construction. IEEE Transactions Inf. Theory, IT, Vol. 44 (1998), No. 6, p. 744-765. 
[18] Vucetic, B., Yuan, J.: Space-Time Coding. John Wiley \& Sons, 2003.

[19] Zajic, A., Stuber, G.: A Space-Time Code Design for Partial-Response CPM: Diversity Order and Coding Gain. IEEE ICC, 2007.

[20] Zhang, X., Fitz, M. P.: Space-Time Code Design with Continuous Phase Modulation. IEEEJ. Sel. Areas Communication, Vol. 21 (2003), No. 5, p. 783-792.

Miroslav Hekrdla

e-mail: hekrdm2@fel.cvut.cz

Department of Radioelectronics

Czech Technical University in Prague

Faculty of Electrical Engineering

Technická 2

166 27, Prague 6, Czech Republic 